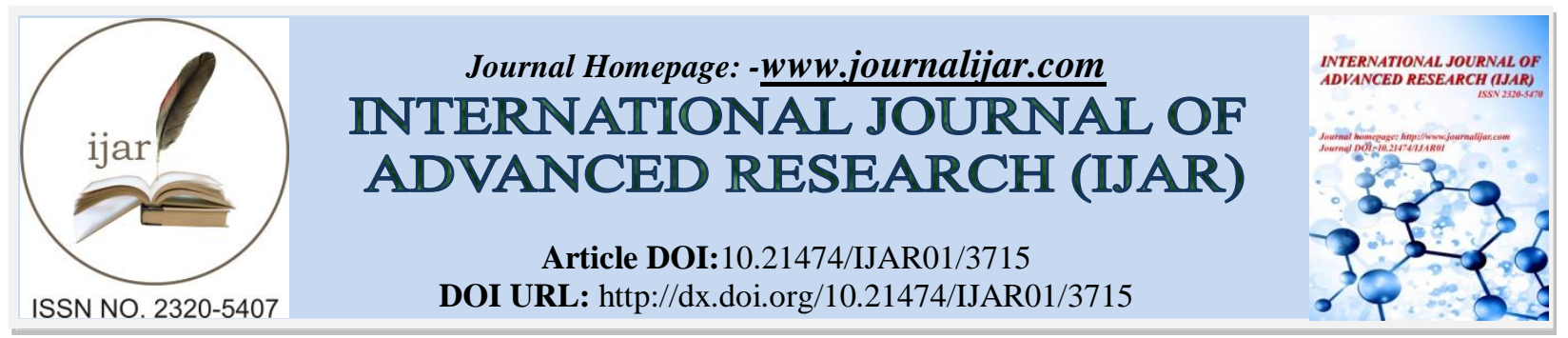

RESEARCH ARTICLE

\title{
HYDROTHERMALISM ASSOCIETED TO THE UPPER VISEAN BASIC MAGMATIC ROCKS OF MACHRAA BEN ABBOU BASIN. (NORTHERN REHAMNA-WESTERN MESETA- MOROCCO).
}

Mariame Kholaiq, Najib Saber, Ghalem Zahour And El Mahdi Ben Sayah.

Laboratory of Geodinamics of Old Belts, Department of Geology, Faculty of Sciences Ben M'Sik, Hassan II University of Casablanca, Morocco.

\section{Manuscript Info}

............................

Manuscript History

Received: 15 January 2017

Final Accepted: 01 February 2017

Published: March 2017

Key words:-

bassin de Mechraa Ben Abbou, Rehamna, Maroc, viséen supérieur, roches magmatiques basiques, altération hydrothermale.

\begin{abstract}
Le bassin de Mechraa Ben Abbou (MBA) (Rehamna-Maroc) a connu au Viséen supérieur une intense activité magmatique basique.

L'analyse structurale par des coupes réalisées sur le terrain a montré que ces roches se mettent en place sous forme de sills. Ces sills suivent en concordance les ondulations déssinées par le plissement principal cartographique du synclinal de la Gada Jennabia. La minéralogie des différents facies identifiés reste comparable avec les minéraux principaux, plagioclases et clinopyroxènes. Ce sont des gabbros à texture grenue, des dolérites à textures ophtique, sub ophitique ou intersertale et des basaltes à texture microlitique porphyrique vacuolaire.

Dans ces roches l'altération se manifeste à l'échelle de l'affleurement par des fillonnets de calcite ou de quartz et au niveau des lames minces par des processus de séricitisation, de chloritisation, de carbonatation, de silicification, d'ouralitisation et d'oxydation. Ces processus d'altération peuvent etre liés à la circulation de fluides hydrothermaux syn ou post-magmatique.

Les diagrammes géochimiques en éléments majeurs en fonction des pertes au feu ont permis de qualifier et de quantifier les effets de l'altération. La confrontation des données de terrain de point de vue structural avec les données microscopiques et géochimiques permettent de caler dans le temps les phénomènes d'altérations observés qui correspondent aux phases tectoniques hercyniennes.
\end{abstract}

Copy Right, IJAR, 2017,. All rights reserved.

\section{Introduction:-}

Le magmatisme lié à l'orogenèse hercynienne au niveau de la Meseta marocaine a fait l'objet de plusieurs travaux de recherche (Jenny, 1974 ; Huvelin, 1977 ; Lagarde, 1987 ; Kharbouch, 1982 ; Kharbouch, 1994 ; Aarab, 1995 ; Essaifi et al., 1995 ; Mrini et al., 1992 ; Remmal, 2000 ; Remmal et al., 1997 ; Roddaz, 2000 ; Ben Abbou, 2001 ; Ben Abbou et al., 2001 ; Kholaiq et al, 2015b). Ce magmatisme est associé aux séries sédimentaires et métamorphiques du Carbonifère terminal.

Corresponding Author:-Mariame Kholaiq. Address:-LCA, Department of Geology, Faculty of Sciences Ben M'Sik, Hassan II University of Casablanca, Morocco. 
Dans le massif des Rehamna septentrionaux, le magmatisme préorogénique est impliqué dans le plissement hercynien d'âge Namuro- Westphalien selon Gigout, (1951). Il s'agit de dolérites et de gabbros sous forme de sills dans la série visèenne et de dykes dans la série du Dévonien inférieur.

Jenny, (1974) et Michard, (1976), signalent l'existence d'ophites et de dolérites en sills et dykes à Mechraa Ben Abbou. C'est ainsi que Jenny, (1974) à travers une coupe réalisée à Bled El Mekrech reprise par Saber, (1989) montre l'existence de sills de puissance métrique séparés par des minces couches métriques de sédiments sur le flanc Est de la Gada Jennabia d'âge Viséen supérieur.

Kharbouch, (1994) a distingué dans les Rehamna septentrionaux deux ensembles magmatiques :

- Un ensemble préorogénique formé par des roches hypovolcaniques et volcaniques ;

- $\quad$ Un ensemble post orogénique formé par des coulées et par des filons microdioritiques.

Elle conclue que les laves sont basiques et que les sills sont constitués de gabbros et de dolérites et que les intrusions sont plus importantes que les faciès effusifs. Les seuls pyroxènes analysés dans l'ensemble magmatique de barrage El Massira révèlent une tendance alcaline. Alors que Remmal et al., (1997) attribuent un caractère tholéitique continental au magmatisme des Rehamna occidentales. Ils signalent que les roches magmatiques sont surtout des dolérites et des gabbros sous forme de laccolites ou de filons sous-marins présentant des pillow-lavas.

Pour El Kamel, (2002)à Mechraa Ben Abbou les intrusions magmatiques sont effusives et intrusives de types alcalin à tholéitique dont leur mise en place est régie par une tectonique distensive attestée par la présence de failles normales syn-sédimentaires.

Les travaux récents Kholaiq et al, (2015b) distiguent au niveau du synclinal de la Gada Janabia des gabbros, des dolérites etde laves basaltiques dont les analyses géochimiques les attribuent à une série tholeitique orogénique continentale (Kholaiq et al., 2016).

\section{Cadre géographique et géologique:-}

Le massif hercynien des Rehamna se situe à $80 \mathrm{Km}$ au Nord de Marrakech et à environ $150 \mathrm{~km}$ au Sud de Casablanca (fig.1). Le bassin de Mechraa Ben Abbou (MBA), objet de notre etude, constitue la partie septentrionale de ce massif.

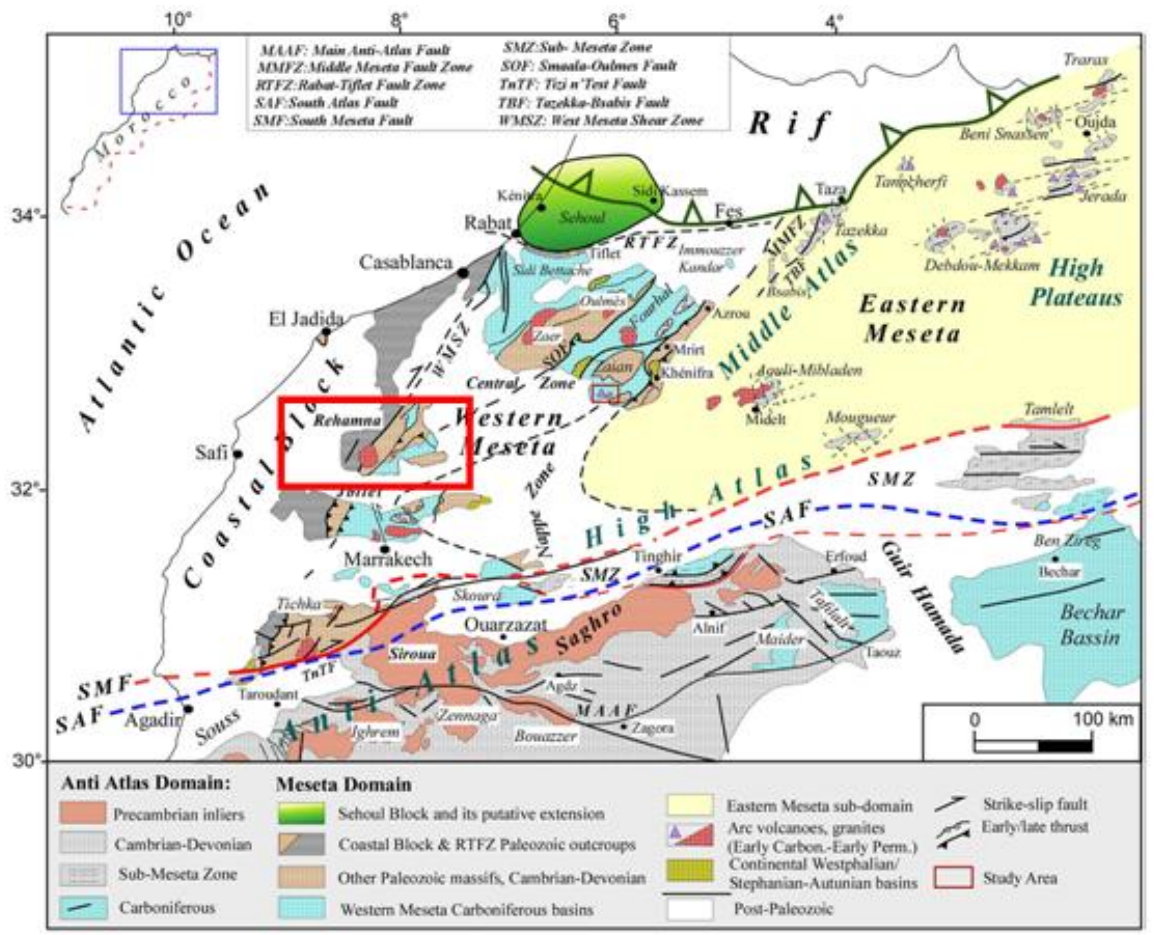

Fig. 1:- Situation du massif des Rehamna dans laMeseta occidentale marocaine (Ntarmouchant et al., 2016). 
Le bassin de Mechraa Ben Abou montre des terrains paléozoïques allant du Dévonien inférieur au Viséen supérieur. Ces terrains présentent une diversification des faciès allant des détritiques, aux carbonatés et aux pélagiques, contemporains de la mise en place des roches magmatiquesbasiques (gabbros, dolérites et basaltes).

L'ensemble est structuré par la phase paroxysmale hercynienne sous forme d'un synclinal (Fig.2) (synclinal de la Gada Jennabia).

Les secteurs de Bled El Mekrech (coupe AB) et Cheikh El Basri (coupe CD) situés sur le flanc Est, le secteur de Lala El Gara (coupe EF) qui constitue la terminaison péri-synclinale et le secteur de Leffaid (coupe GH) situé sur le flanc Ouest, sont les quatre sites qui permettront d'approcher le magmatisme dans le bassin (Fig.2).

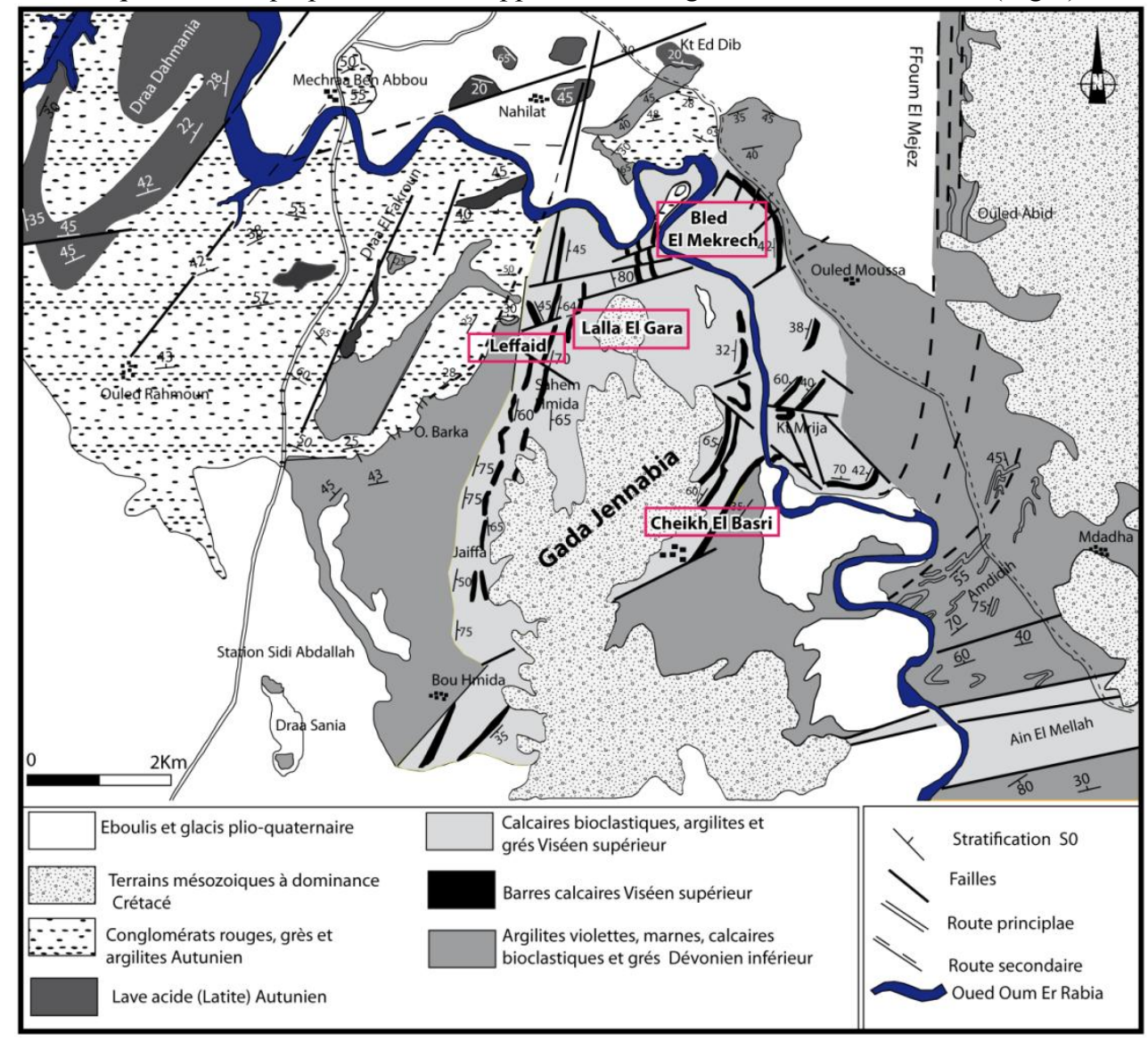

Fig. 2:- Répartition des affleurements de roches magmatiques basiques dans le synclinal de la Gada Jennabia (Kholaiq et al, 2015a)

\section{Caractérisation structurale et pétrographique des roches magmatiques:-}

Les données pétrographiques ont été obtenues à partir de l'étude d'une vingtaine d'échantillons considérés comme représentatifs. Des lames minces ont été observées au microscope pour déterminer les textures et les associations minéralogiques de ces roches. De point de vue mode de gisement, l'ensemble des roches magmatiques forment des sills et les coulées de laves parallèles à l'encaissant immédiat d'âge Viséen supérieur probable. Ainsi, leurs caractères relativement résistant à l'érosion et à l'altération mettent les gabbros et les dolérites en relief au sein des schistes. Les roches sont généralement de couleur vert olive quant elles sont fraiches, et prennent une couleur sombre par altération.

Mais avant d'étudier ses roches, et pour mieux cerner le mode de la mise en place des roches magmatiques nous avons réalisé plusieurs coupes sur le terrain. 


\section{Coupe de Bled el Mekrech:-}

Relation roche magmatique - encaissant:-

Une coupe réalisée sur le flanc Est de la Gada Jennabia, orientée ESE-WNW (fig.3), révèle la présence des plis dans les terrains d'âge Dévonien inférieur. Ces plis de taille métriques sont tous de type isopaques dont l'orientation des axes varie de N90 à N125 avec un prolongement axial de $25^{\circ}$ à $30^{\circ}$ vers l'Ouest.

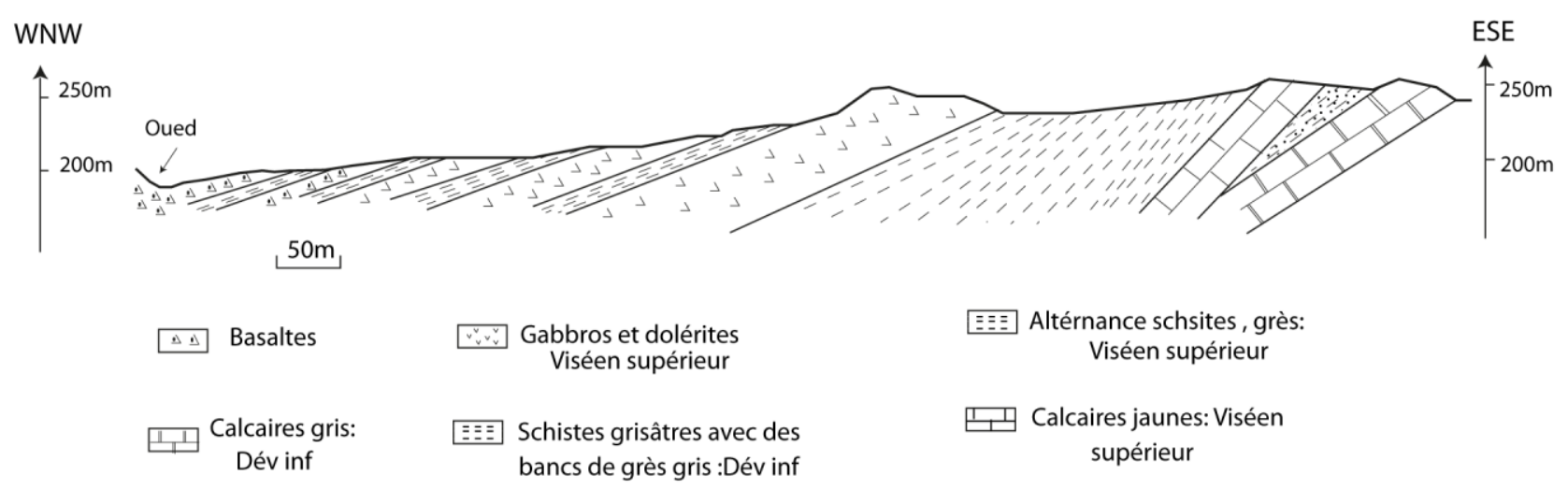

Fig.3:- Coupe géologique dans les terrains Dévono-Viséens de Bled El Mekrech.

Sur le flanc Sud, la direction des couches varie de N100 à N105 avec un pendage de 45 à $40^{\circ}$ vers le Sud. Sur l'autre flanc la direction est de N75 avec un pendage de $25^{\circ}$ vers le Nord. Au niveau de la charniere la stratification est orientée N145 avec un pendage de $30^{\circ}$ vers l'Ouest. La schistosité est surtout visible dans les bancs incompétents schisteux. Elle est sub-verticale avec une orientation N15 à N20. Cette schistosité parfois subit des virgationsillustrant des zones de cisaillement à l'échelle de l'affleurement orientées N120-N130 senestres.

La deuxième barre calcaire d'âge Viséen supérieur montre une stratification orientée N160 avec un pendage de $30^{\circ}$ dans laquelle on observe des plis SE. Au niveau de la surface structurale de cette barre on rencontre des fentes de tension remplis par la calcite de direction N55 et N160 et un pendage de 55 vers l'Est parfois en échelons matérialisant des zones de cisaillement.

La barre de Viséen supérieur est suivie par une alternance de schistes et grès dont l'orientation est la même que la barre calcaire de Viséen supérieur avec un pendage de $55^{\circ}$ vers l'Ouest.

Dans les gabbros et les dolérites, on trouve des miroirs de failles N170 $55^{\circ}$ vers l'Est ainsi que des cassures de même direction remplis par la calcite. Ces cassures sont parfois plissées. On trouve aussi des filons pluri-métriques N 130 sub-verticaux, remplis par du quartz. Le contact encaissant - gabbros et dolérites est souvent accompagné d'une schistosité de fracture sub verticale N110 à N120. (Fig. 4). Par endroits ce contact prend un aspect bréchifié. Plus on se dirige vers l'Oued c'est-à-dire vers le cœur du synclinal le pendage des couches devient de plus en plus faible pour atteindre des valeurs très faibles $\left(20^{\circ}\right.$ vers l'Ouest $)$.
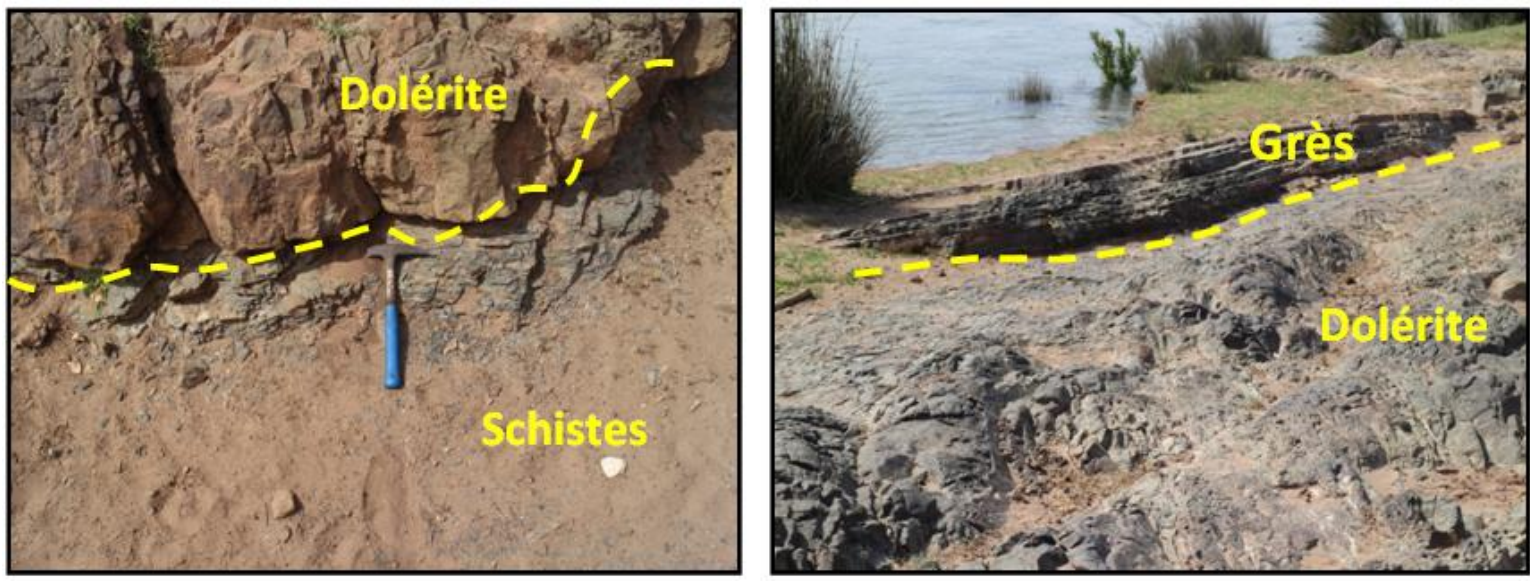

Fig. 4:- Photos de terrain montrant la relation entre les dolérites et les roches encaissantes 


\section{Pétrographie:-}

L'étude microscopique des roches magmatiques à Bled El Mekrech (Fig.5), nous a permis de mettre en évidence un faciès gabbroique. Lesgabbros de couleur vert clair à vert foncé développent une variété de textures, grenue, microgrenue, et doléritique. Dans les gabbros à texture grenue, lesassociations minéralogiques montrent un gabbro à pyroxène et un gabbroordinaire sans pyroxène. Les gabbros à pyroxène (Fig.5,A) montrent une composition minéralogique à plagioclases (30 à 40\%), de type labrador ou les macles polysynthétiques sont assez fréquentes. La taille des lattes est comprise entre 2 et $5 \mathrm{~mm}$. Ces cristaux développent une altération en calcite et en chlorite. Le clinopyroxène 40 à 50\%, est le minéral le plus abondant après le plagioclase, et forme des plages xénomorphes très fissurées. Il montre des transformations en granules d'oxydes noirâtres, en chlorite, et/ou en calcite.

Vers les bordures figées et à l'approche du contact avec l'encaissant, les gabbros ordinaires (Fig.5, B) montrent une texture microgrenue ou les phénocristaux sont représentés par des plagioclases millimétriques et des transformations surtout en calcite. Dans le faciès à texture doléritique (Fig.5, C et $\mathrm{D}$ ), les plagioclases et les clinopyroxènes constituent la paragenèseprimaire (60\% à 70\%) et la calcite, la chlorite et les oxydes de fer constituent la paragenèse secondaire. Le plagioclase de type labrador, cristallise en lattes maclées sub-tabulaires de 0,4 à $4 \mathrm{~mm}$ de long. Ces dernières sont en association intersertale triangulaire renfermant des clinopyroxènes. Parfois, les plagioclases se présentent sous forme de baguettes à l'intérieur des clinopyroxènes, conférant à la roche une texture ophitique.
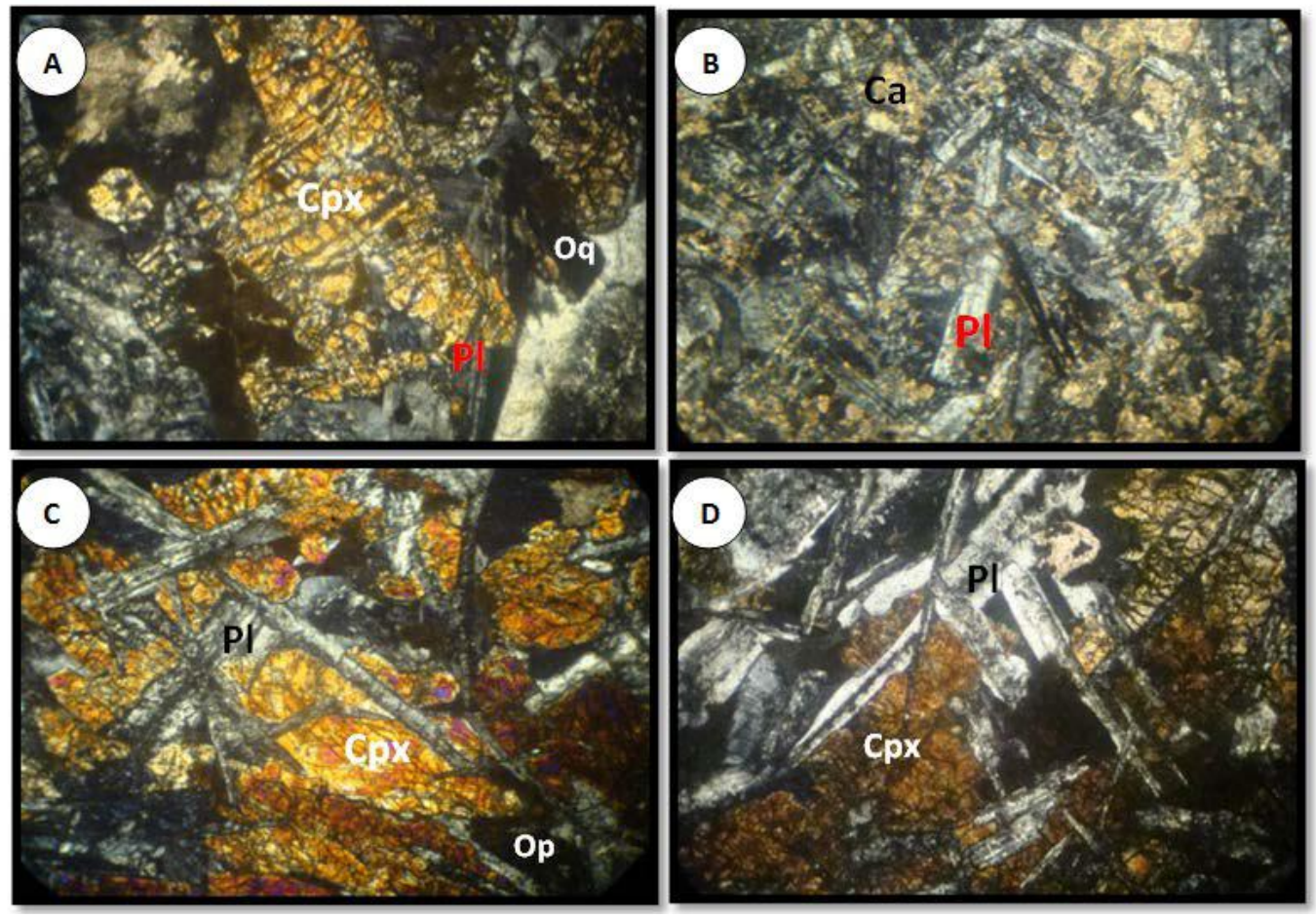

Fig .5:- Photographie des associations minérales des roches magmatiques basiques de Bled ElMekrech.

A : gabbro à pyroxène au coeur du sill. B : gabbro ordinaire des bordures figées. $C$ et $D$ : dolérite à texture intersertale et sub-ophitique. Pl: Plagioclase. Cpx : Clinopyroxène. Ca : Calcite.

Op : Opaque $\left(\mathrm{Gr}^{*} 100\right)$

\section{Coupe de Cheikh El Basri :}

\section{Relation roches magmatique - encaissant:-}

C'est une coupe E-W réalisée dans les terrains géologiques de Cheikh El Basri (fig.6), les formations détritiques et carbonatées recoupées par des sills des roches magmatiques basiques d'âge Viséen supérieur, forment une structure monoclinale à pendage vers l'Ouest. Cette structure est surmontée par une couverture tabulaire d'âge Mésozoïque en discordance angulaire. 
A la base, on trouve des schistes caractérisés par une schistosité de fracture. Ces schistes sont intercalés par des bancs gréseux, l'ensemble est orienté N40 avec un pendage vers l'Ouest. Les filons gabbroiques sont intercalés d'une façon concordante avec une barre calcaire orientée N50 avec un pendage de $70^{\circ}$ vers le Nord. Au dessus de cette barre on trouve un deuxième affleurement gabbroique qui à toujours la même direction que l'encaissant. Par endroit, le contact immédiat est souvent caché par des éboulis qui rendent difficile de toucher et préciser la nature de la limite encaissant- gabbro. Les schistes qui viennent après sont perturbés (aspect chaotique) assimilables à des brèches tectoniques, ce qui confirme un passage de faille. Le haut de ces schistes est caractérisé par des passés gréseux de couleur grisâtre à noirâtres dite cornés orienté N40 avec un pendage vers l'Ouest.

Sur les schistes un filon gabbroique est de couleur rougeâtre parfaitement parallèle avec la stratification témoigne une autre fois une mise en place sous forme de sill stratiforme.

Sur ces gabbros repose une barre calcaire N50 avec un pendage de 70 à $60^{\circ}$ vers l'Ouest. Avec cette barre s'affine le Viséen supérieur et commence le continentale intercalaire. Il est formé essentiellement par des grès, calcaires et des argiles rouges dont la stratification est orientée N40. Dans la partie sommitale, on trouve une alternance des barres calcaires et des marnes jaunâtres constituent une couverture sub-tabulaire.

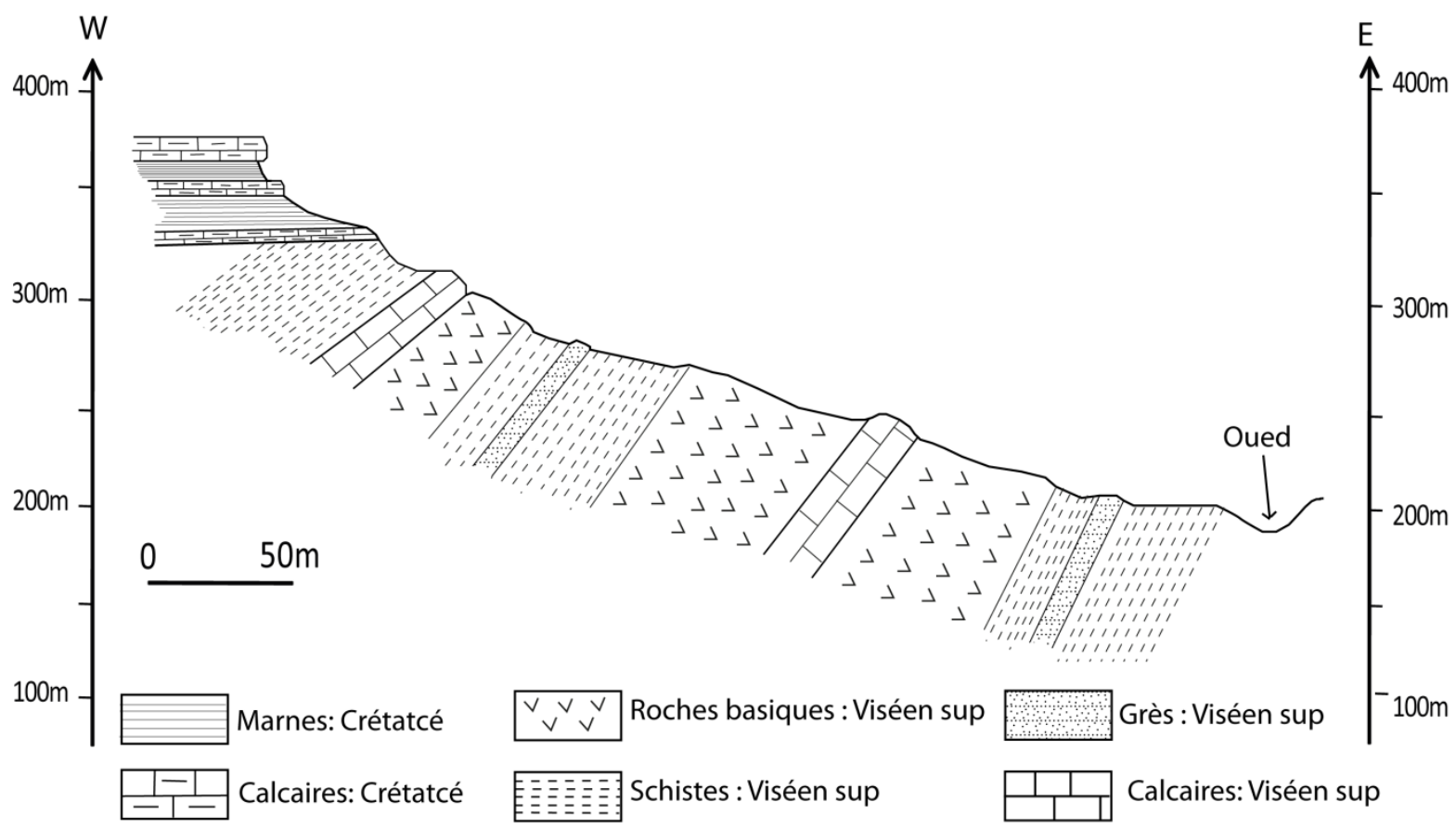

Fig. 6:-Coupe géologique E-W à travers les terrains Viséens supérieur à Cheikh El Basri

\section{Pétrographie:-}

Ce massif constitue l'un des principaux corps magmatiques des Rehamna septentrionaux. Dans les travaux antérieurs, il n'a jamais faitl'objet d'étude pétrographique. Il s'agit de dolérite de couleur rouge surpatine et sur cassure fraiche. Au microscope la texture est intersertale \pm ophitique et/ou sub-ophitique (Fig.7). La composition minéralogique estmarquée par une proportion en plagioclase quasi égale à celle des minérauxferromagnésiens représentés par des clinopyroxènes. Le plagioclase enphénocristaux cristallise en premier. Il est de type labrador avec des maclespolysynthétiques. Il est fréquemment fracturé mais rarement altéré. Leclinopyroxène qui cristallise en plages xénomorphes dans une texture de type ophitique à sub- ophitique où les lattes de plagioclases sont incluestotalement ou partiellement dans les plages de clinopyroxène. 

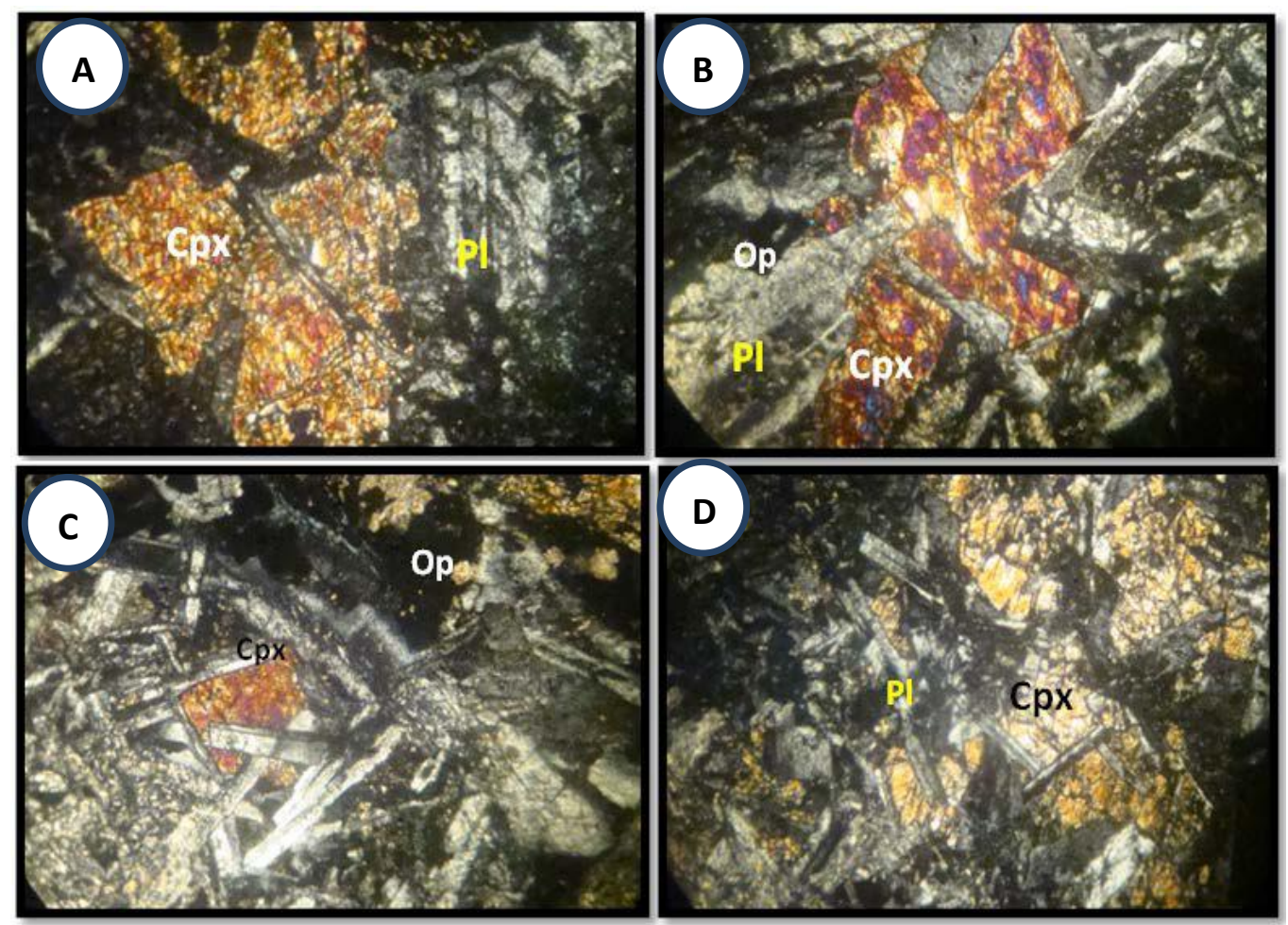

Fig .7:-Photographie des associations minérales des roches magmatiques basiques deCheikh El Basri. A et $B$ : Dolérite à texture inter tale, ophitique et sub-ohitique. C : Dolérite à texture inter tale et sub-ophitique. D: Dolérite à texture inter tale, ophitique et subohitique. $\mathrm{Pl}$ : Plagioclase. Cpx : Clinopyroxène. Ca : Calcite. Op : Opaque (Gr*100)

\section{La coupe de lala El Gara:-}

\section{Relation roches magmatique - encaissant:-}

La coupe W-E réalisée au niveau de la terminaison périsynclinale de la Gada Jennabia (Lala El Gara) révèle (fig.8) : Une série schisto- gréseuse où s'intercale des gabbros sous forme de sills. La stratification aux limites des gabbros est orienté N150 avec un pendage de $70^{\circ}$ vers le Nord.

Dans cette formation on a pu mettre en évidence des plis dont le cœur présente une schistosité de plan axial N20 N30 sub- vertical donnant un aspect en frites aux schistes lorsqu'elle coupe la stratification.

En allant vers l'Ouest, les sills stratiformes suivent le plissement des schistes pour former des synclinaux et des anticlinaux de direction N20 - N30, le pendage sur les flancs est généralement de l'ordre de $50^{\circ}$ vers l'Est et de $30^{\circ}$ vers l'Ouest. Ces plis apparaissent dans des couloirs limités par des failles verticales.

Le plissement des sills suivant les ondulations observées surtout au niveau de la terminaison périsynclinale de Lala El Gara confirme une mise en place syn phase paroxysmale (fig 9 et 10).

Lala El Gara au sommet présente une structure synclinale dont les pendages aux flancs sont faibles, soit vers l'Est ou soit vers l'Ouest. Elle est bien marquée par les coulées basaltiques interstratifiées dans une formation schisteuse. 


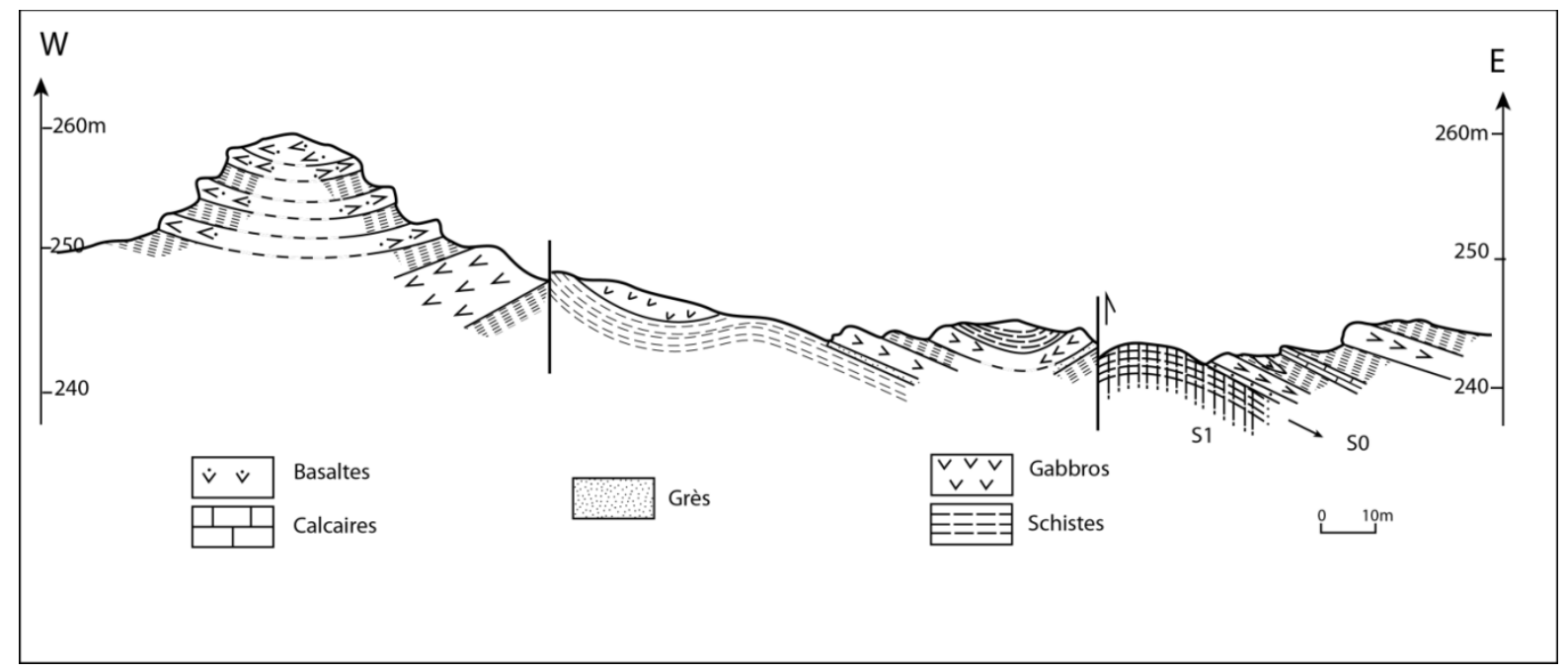

Fig.8:- Coupe géologique dans les terrains Viséens de Lala El Gara.
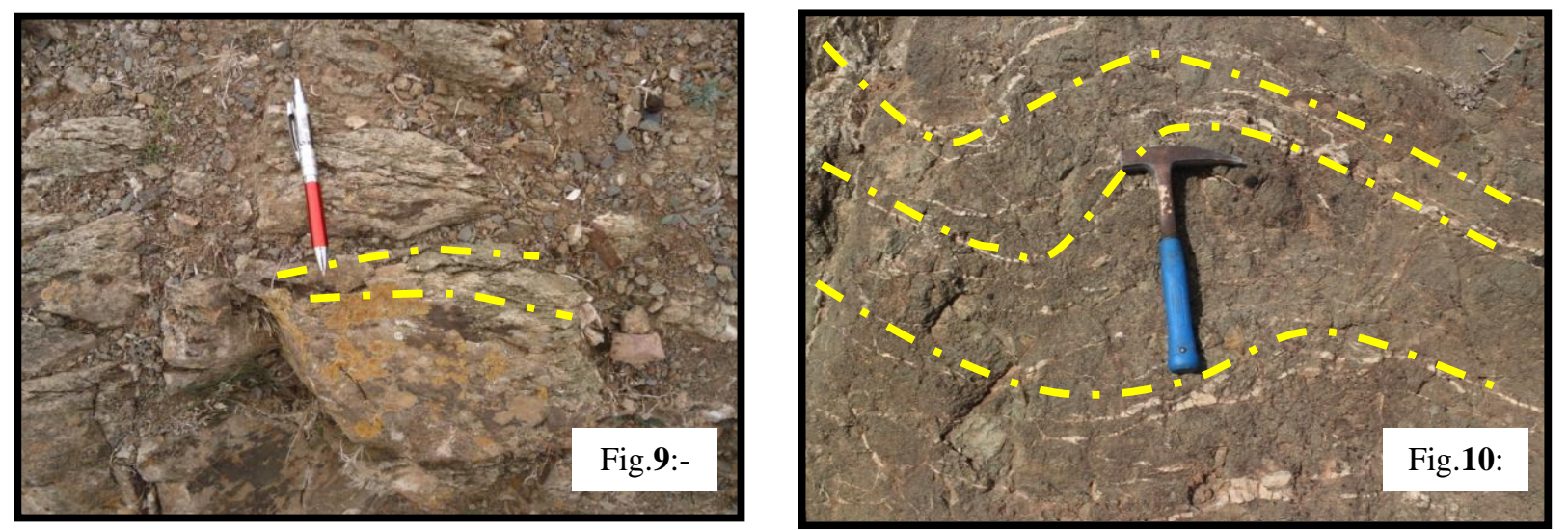

Fig.9:- Photo montrant un débit schisteux dansles roches magmatiques basiques.

Fig.10:- Photo montrant le plissement des veines calcitiques au sein des gabbros.

\section{Pétrographie:-}

L'étude pétrographique des roches magmatiques de Lala El Gara montre qu'il s'agit de deux ensembles : un ensemble doléritique et un ensemble basaltique. L'ensemble doléritique (Fig.11, A et B) a une composition minéralogique semblable à celle de Bled El Mekrech et de Cheikh El Basri, mais s'en diffère par l'abondance des plagioclases. Le plagioclase constitue le minéral cardinal, de type labrador sous forme des cristaux automorphes avec des macles polysynthétiques. Le clinopyroxène cristallise en plages qui peuvent englober totalement ou partiellement des cristaux de plagioclases formant une texture ophitique à sub-ophitique.

L'ensemble basaltique est reconnaissable grâce à la présence de vacuoles centimétriques, la roche est de couleur grise à verte olive. A l'échelle de la lame mince, la roche a une texture microlitique porphyrique vacuolaire (Fig.11, $\mathrm{C}$ et $\mathrm{D})$. La paragenèse primaire est constituée essentiellement par des cristaux de plagioclases de type labrador, sous forme de baguettes allongées de taille varient de 0,05 à $4 \mathrm{~mm}$, qui constituent $60 \%$ à $70 \%$ du volume total de la roche. La macle polysynthétique est bien distincte. Ces cristaux sont emballés dans un fond vitreux (mésostase), qui représente $30 \%$ à $40 \%$ de la roche. Celle-ci renferme des vacuoles à remplissage calcitique, dont certaines montrent un remplissage séquentiel avec de la chlorite au centre et la calcite en bordures. Ce caractère traduit, soit la présence de deux phases d'hydrothermalisme liées respectivement à deux phases orogéniques, soit l'existence de deux stades d'hydrothermalisme tardi à post-magmatiques d'une même phase tectonique dont l'un est précoce et l'autre est tardif. Les vacuoles peuvent être isolées ou communicantes avec deux ou plusieurs individus. 

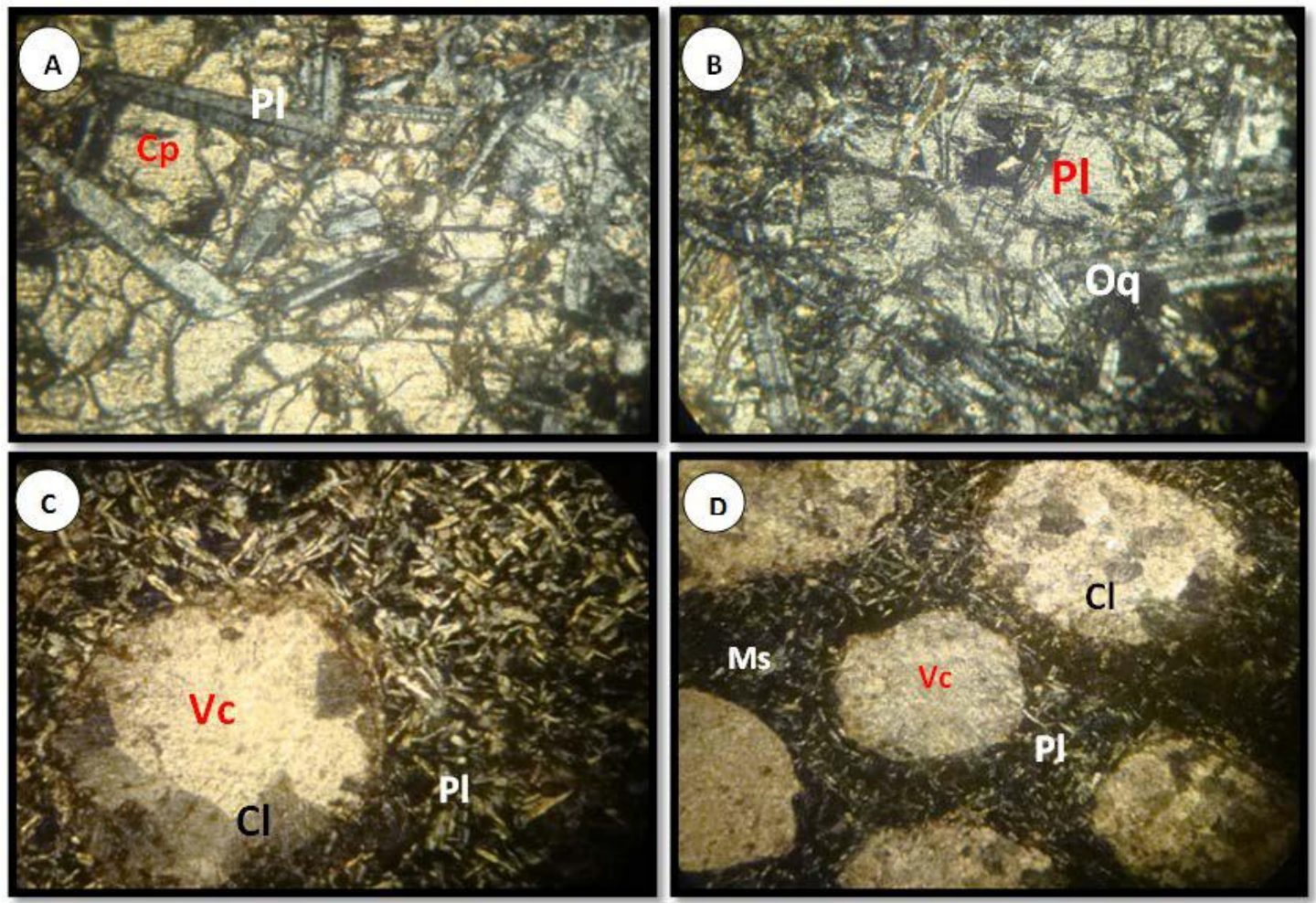

Fig.11:- Photographies des dolérites et des basaltes de Lala El Gara.

A : Dolérite à textureophitique et sub-ophitique (centre du sill). B : Dolérite des bordures figées. $C$ et $D$ : Basaltes porphyriques microlitiques vacuolaire $\mathrm{Pl}$ : Plagioclases, Cpx: Clinopyroxènes , Ca: Calcites

, Oq: Opaques, Vc: Vacuoles Ms : Mésostase (Gr*100)

\section{A Leffaid:-}

Relation roches magmatique - encaissant:-

Il s'agit d'une coupe E-W (Fig. 12), réalisée dans les terrains viséens de Leffaid (flanc Ouest du synclinal de la Gada Jennabia. En se basant sur les relations entre les différents faciès, une succession lithostratigraphique a pu être reconstituée, cette dernière comprend de bas en haut (de l'Ouest à l'Est) :

Des schistes grisâtres orientés N30 avec un pendage de 40 à 30 vers l'Est, suivi par une barre calcaire orientée N160 avec un pendage de 40 vers l'Es. Au niveau de la surface structurale on trouve des gradins matérialisés par des failles N80 portant des stries à aspect boueux, cette barre est suivie par une alternance de schistes et de grès dont l'orientation est N30 avec un pendage de 30vers Est.

Après une deuxième barre calcaire un peu pentée que la première mais qui garde la même direction et qui est toujours vers l'Est, des filons gabbroiques sont intercalés d'une façon concordante avec des schistes et des grès (fig 13), sur ces roches magmatique on remarque un débit orienté N30 et des fractures orientée N80 remplis de calcites et de quart. Une alternance de coulés basaltique et des schistes coiffent la série visèenne.

Les terrains du Viséen supérieur sont couverts en partie par une série tabulaire d'âge mésozoïque sur lesquels elle repose en discordance. 


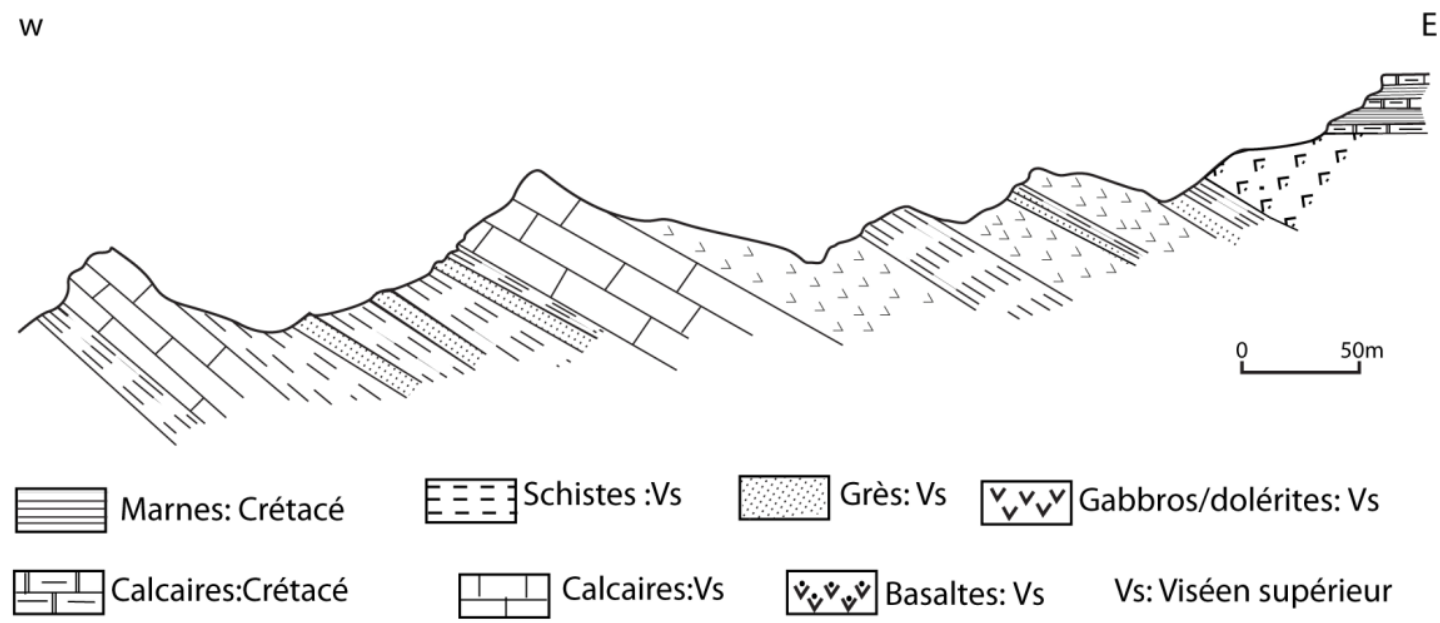

Fig. 12:- Coupe géologique à travers les terrains Viséens supérieur de Leffaid
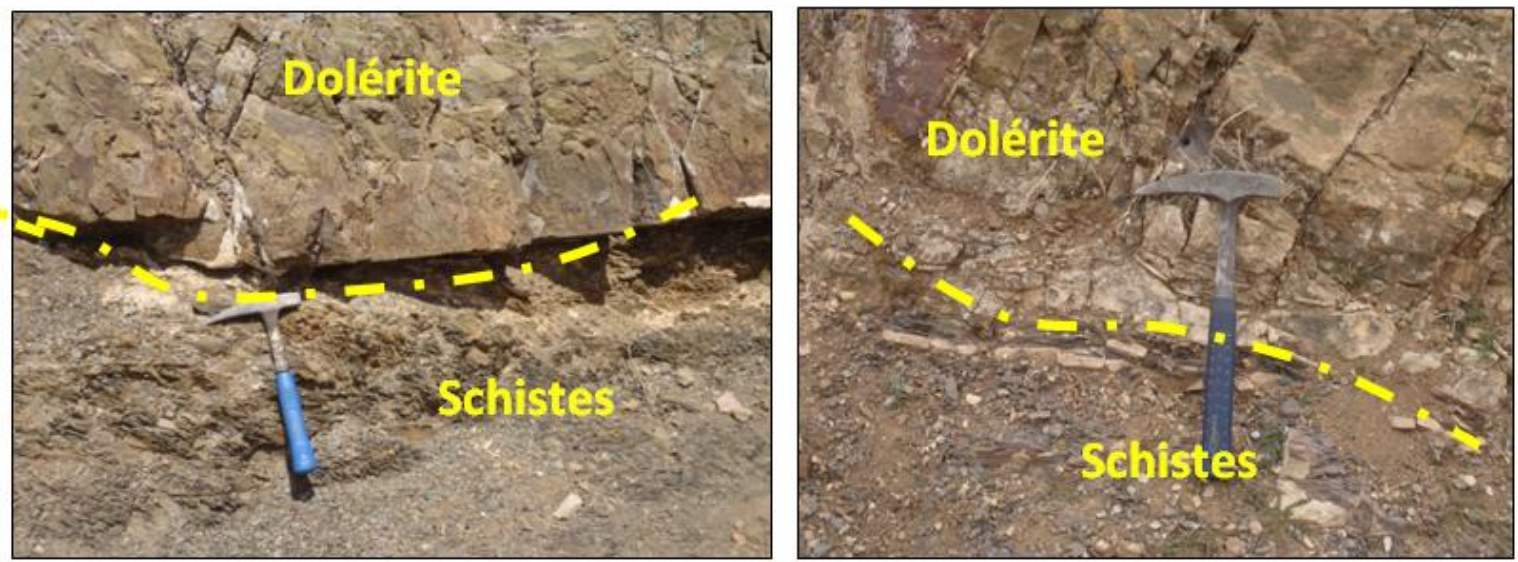

Fig. 13:- Photo montrant la relation entre les dolérites et les roches encaissantes

\section{Pétrographie:-}

L'étude microscopique des roches magmatiques dans la région de Laffaid nous a permis de mettre en évidence deux ensembles : un ensemble doléritique (Fig 14, A et B) et un autre basaltique (Fig 14, C et D).

L'ensemble doléritique est caractérisé par une couleur vert olive sous forme de sills. Il montre à l'oeil nu des plagioclases et des clinopyroxènes. Microscopiquement, les épontes de ces sillssont caractérisées par une texture microcristalline. Vers le coeur, la texture évolue est et devient intersertale, ophitique et/ou sub-ophitique. La paragenèse minérale est constituée essentiellement par des plagioclases, des clinopyroxènes, des opaques et accessoirement de la calcite, de la séricite et de la chlorite.

Le plagioclase est en général séricitisé. Il représente $40 \%$ à $50 \%$ des minéraux de la roche. Il s'agit en général de labrador sous forme des cristaux automorphes avec des macles polysynthétiques. Le clinopyroxène, le plus souvent transformé en chlorite et/ou en calcite, représente 30\% à 50\% des minéraux de la roche. Il est de type clinopyroxène formant avec les plagioclases une texture doléritique de type ophitique et sub-ophitique. Le clinopyroxène cristallise sous forme des plages xéno-morphes très fissuré où on observe des oxydes de fer.

Les transformations secondaires sont, en plus de le chlorite, les oxydes de fer et la calcite. Les minéraux opaques sont souvent automorphes et abondants dans les roches. 

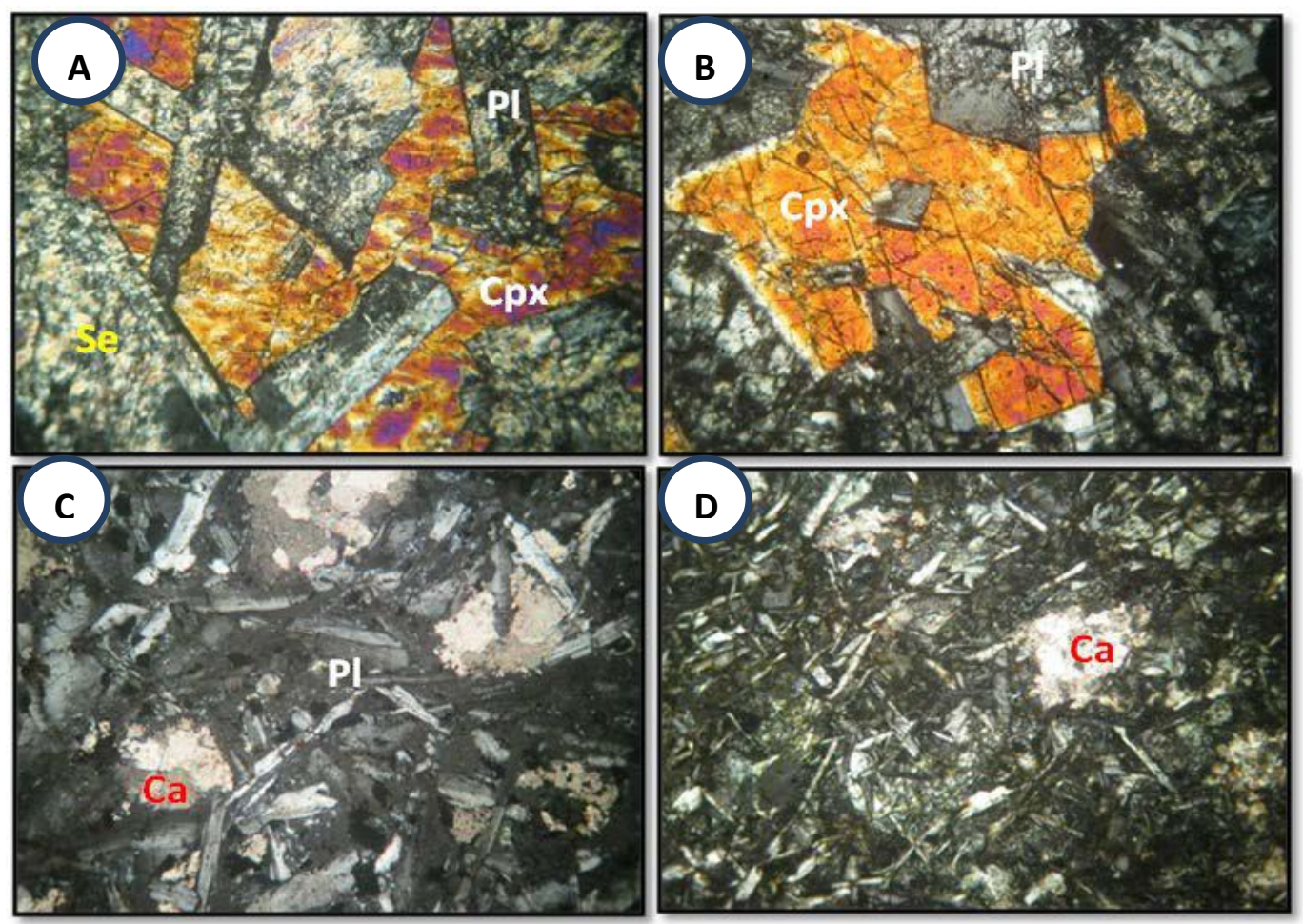

Fig 14:- A, B : Dolérites à texture inter tale, ophitique et sub-ohitique. : Dolérite à texture intertale et sub-ophitique. $\mathrm{C}$ et $\mathrm{D}$ : Basaltes porphyriques microlitiques vacuolaire.

Pl :Plagioclase. Cpx : Clinopyroxène. Ce : Séricite, Ca : Calcite. Op : Opaque (Gr*100)

Il parait que les roches magmatiques des quatre affleurements (Bled El Mekrech, Cheikh El Basri, Lala El Gara, et Leffaid), présentent des minéralogies comparables (Tableau I) a paragenèse primaire des gabrros et des dolérites est caractérisée par une abondance du plagioclase suivi par le clinopyroxène formant des textures ophitiques, subophitiques et intersertales dont la paragenèse secondaire est surtout représentée par des minéraux d'altérations tels que la calcite, la chlorite, et les oxydes de fer.

Les basaltes microlitiques porphyriques vacuolaires de Lala El Gara et Leffaid sont caractérisés par des plagioclases. Les minéraux d'altération sont représentés par la Calcite, la Chlorite et la Séricite. Concernant l'ordre de cristallisation et d'après la relation mutuelleentre les minéraux, on constate la cristallisation d'abord d'opaques, de plagioclase, puis de clinopyroxène (Fig.15).

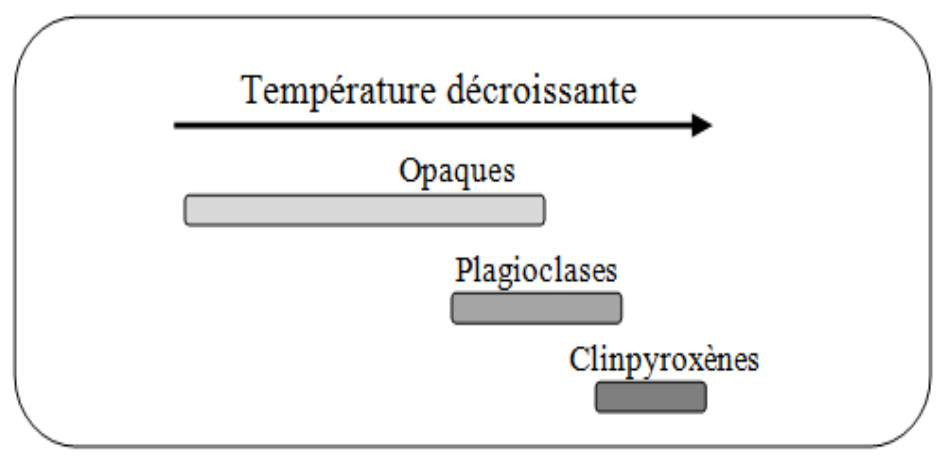

Fig.15:- Schématisation de l'ordre de cristallisation 


\section{Altération des roches magmatiques étudiées:-}

A l'échelle d'affleurement et du banc, Les phénomènes d'altération sont manifestés par des filons de calcites et de quartz. En effet, les roches magmatiques sont affectées par une fracturation plus ou moins intense. Les fractures $(0,5$ à $4 \mathrm{~cm}$ d'ouverture) sont remplies selon les cas de calcite ou d'ankérite.

A Bled El Mekrech, les roches magmatiques sont affectées par deux familles de fractures remplies par la calcite. La première famille est sub verticale orientée N140, alors que la deuxième famille tardive orienté N20. .Parfois, l'aspect réticulé rend difficile l'établissement d'une chronologie relative de leur apparition. Par endroit, les fractures deviennent très serrées et sont remplies par la calcite, qui illustre des amygdales de cisaillement.

Parfois, ces fractures sont plissées indiquant un remplissage anté-déformation. Certaines fractures montrent un remplissage polyphasé (séquentiel), avec cristallisation de la calcite au centre et de l'ankérite en bordures. (fig.16).
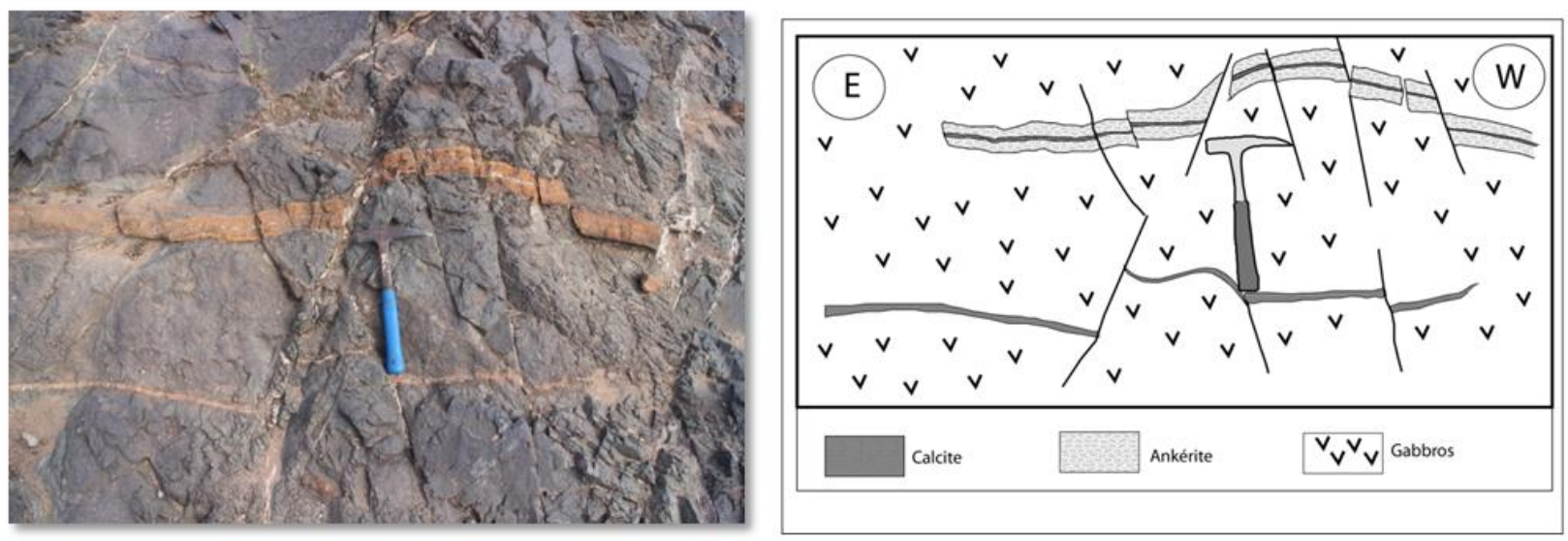

Fig.16:- fractures montrent un remplissage polyphasé

A cheikh El Bassri, les roches magmatiques sont traversées par des fractures N 70 remplies aussi par la calcite, alors que à Lala El Gara, les roches sont affectées par deux familles de fractures, dont la première est orientée N150 sub- verticale, et la deuxième famille est orientée N90 avec un pendage de $50^{\circ}$ vers le Nord.

Microscopiquement, les roches magmatiques des trois zones d'études montrent des transformations en calcites, chlorites, opaques et en quartz rarement au centre des sills très intensément aux bordures figées.

Calcitisation:-

La calcite présente en quantité appréciable dans la majorité des échantillons. Elle représente un produit de transformation et d'altération des minéraux primaires. Elle se rencontre presque dans les roches altérées qui se situent aux bordures des sills. Ainsi Certaines roches sont complètement transformées en calcite. Cette dernière cristallise soit en petites plages, soit sous forme des paillettes et / ou des petites veines qui recoupent les lattes de plagioclases (fig.18.a). Elle cristallise dans les cassures des cristaux de clinopyroxènes et de plagioclases aussi bien qu'à la limite plagioclase-clinopyroxène.Dans les gabbros de Cheikh El Basri, la calcite est associée à l'ankérite où au chlorite. (fig.17et 18.b). 

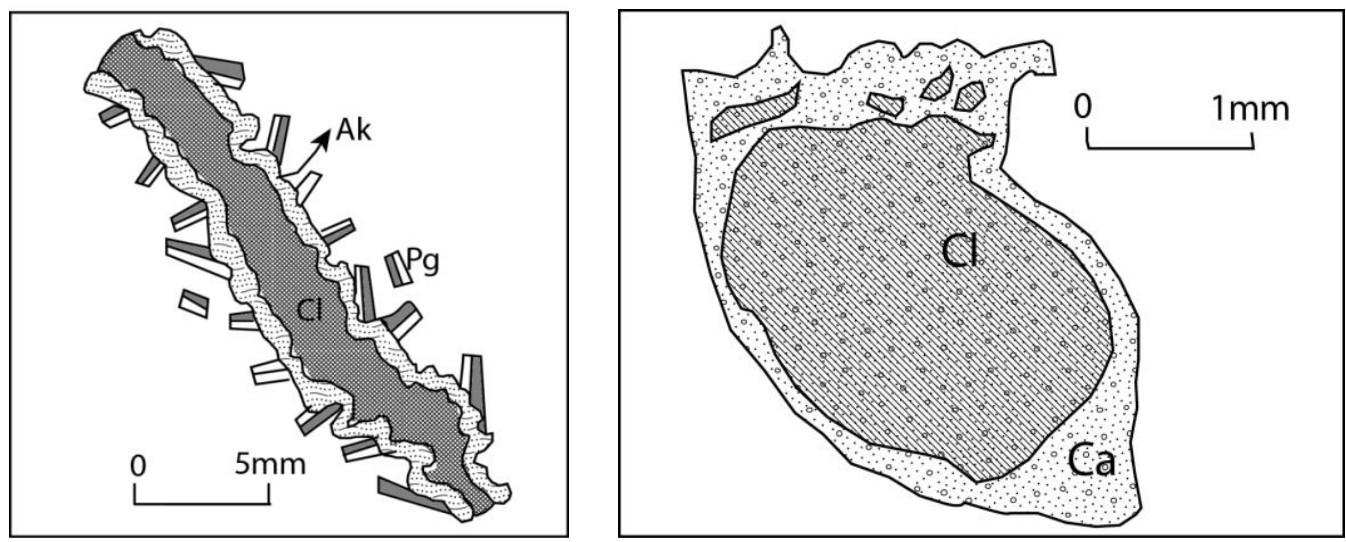

fig. 17:-Altération polyphasée dans les gabbros de Cheikh El Basri

Ak : Ankérite, $\mathbf{C l}$ : Chlorite, $\mathbf{C a}$ : Calcite, Pg : Plagioclase

\section{Chloritisation :-}

La chlorite est un minéral secondaire qui se développe aussi bien dans gabbros que les dolérites. Elle cristallise en petits amas qui se développement aux dépens du clinopyroxène et du plagioclase. Grace à sa couleur verdâtre, elle se reconnait facilement en lame mince. Elle se rencontre associée à d'autres produits de transformation telle que la calcite (fig.18.c).

\section{Oxydation :-}

L'oxyde de fer fait partie des produits d'altération les plus répondus dans toutes les lames altérées. Il se développe au dépend des fractures des minéraux surtout les pyroxènes (fig.18.d).

\section{Ouralitisation:-}

Ouralitisation des pyroxènes est la formation d'amphibole (Trimolites ?) qui cristallise en amas et en plage au dépend des pyroxènes (fig.18.e).

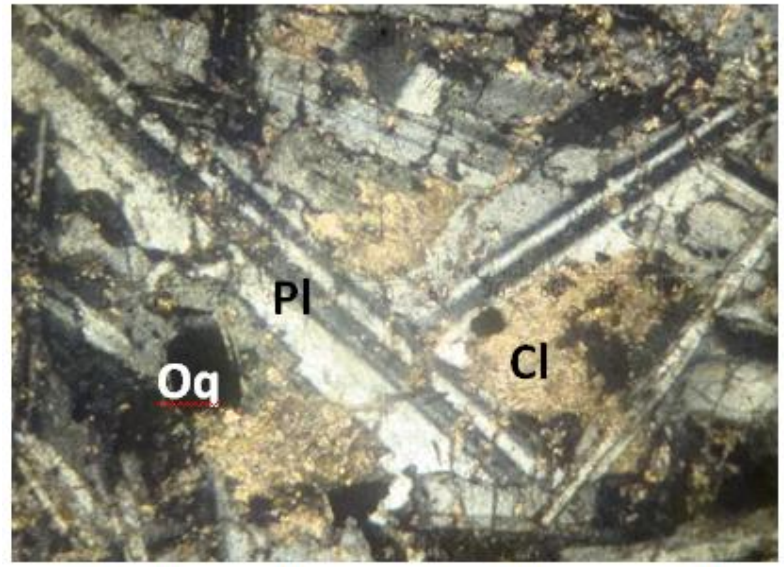

Fig.18.a: Calcite au dépend des plagioclases

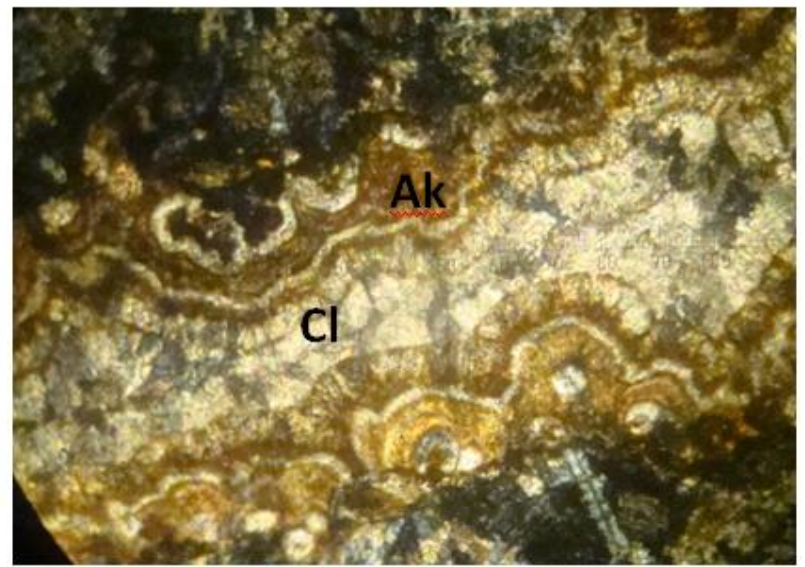

Fig.18.b : Calcite au milieu de l'ankérite 


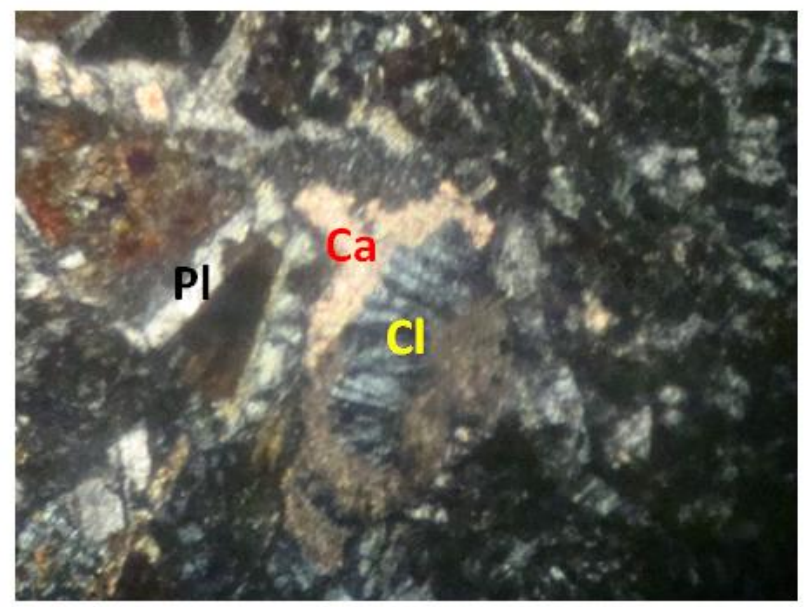

Pl : Plagioclases

Cp : Clinopyroxènes

Ca : Calcites

$\mathrm{Cl}$ : Chlorites

Og: Opaques

Ak : Ankérites

Fig.18.c : Chlorite dans la calcite
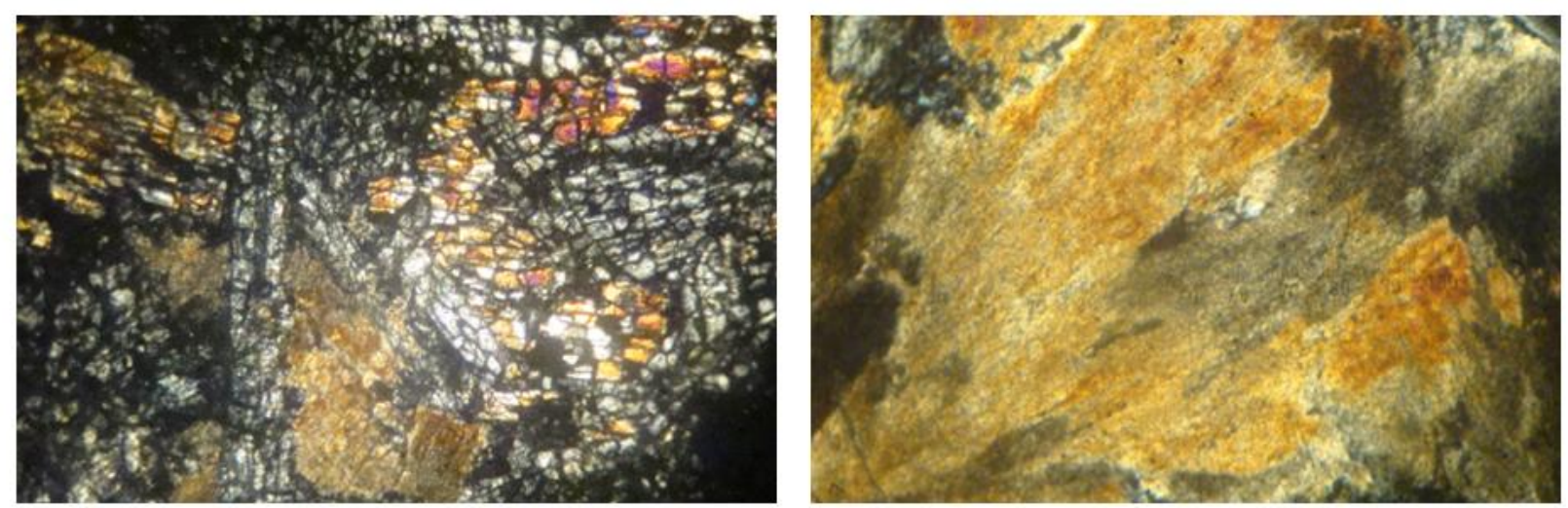

Fig.18.d : Oxyde de fer dans les fractures de pyroxènes Fig.18.e : Amphibole

Fig.18:- lames minces des différents produits d'altérations (LPA) Gr*100

\section{Approche géochimique des effets de l'altération:-}

Les compositions chimiques des roches analysées peuvent être affectées par une altération post-magmatique. En effet, elles montrent des transformations hydrothermales liées aux événements tectono-métamorphiques hercyniens. D'après une lecture directe des compositions chimiques et en particulier les pertes au feu qui traduisent le plus souvent la transformation des phases minéralogiques primaires en phases secondaires hydratées. L'examen des analyses chimiques (tableau) montre que les roches étudiées présentent des pertes au feu variable, de 4,16 à 9,84\%. Les valeurs élevées traduisent une altération importante des minéraux primaires.

Dans ce cas, le diagramme $\left(\mathrm{K}_{2} \mathrm{O}+\mathrm{Na}_{2} \mathrm{O}\right)$ en fonction de $\mathrm{K}_{2} \mathrm{O} /\left(\mathrm{K}_{2} \mathrm{O}+\mathrm{Na}_{2} \mathrm{O}\right)$ de Hughers (1973) (Fig 19)nous semble très important du fait qu'il définit le champ des roches de composition magmatique initiale, le champ des roches enrichies en potassium et celui des roches enrichies en sodium

L'examen de ce diagramme montre que les points représentatifs des quatre affleurements du bassin de MBA gardent l'empreinte des caractères magmatiques originaux comme l'indique leur emplacement dans le spectre des roches ignées

Donc même avec des valeurs élevées des pertes au feu, les roches étudiées gardent leur caractère initiale, on peu dire donc qu'elles ont subissent une altération poste formation 


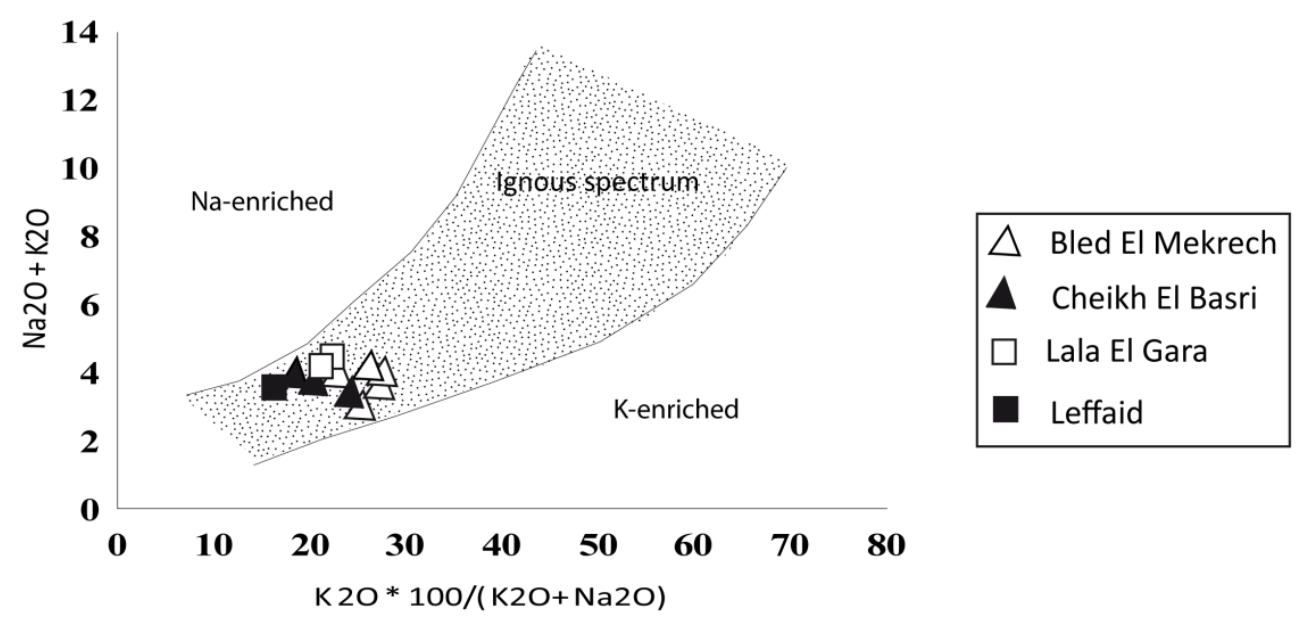

Fig 19:- projection des roches magmatiques basiques étudiées dans le diagramme de Hughes (1973) montrant que ces roches ont une composition magmatique originelle comparable

\section{Diagrammes oxydes pertes au feu:-}

A partir de la lecture des analyses chimiques brutes des roches magmatiques, nous constatons que les pertes au feu sont relativement élevées, les roches magmatiques peuvent en contenir jusqu'à 9,84\%

Les fluctuations des pertes au feu des roches étudiées semblent être liées à la proportion des minéraux hydratés (chlorites, séricites) et la néoformation de la calcite et le quartz dans les cassures, les veines et les vacuoles dans les basaltes, Il est évident que les teneurs en pertes au feu soient proportionnelles au degré d'hydratation que le magma et les minéraux peuvent subir. Ce phénomène qui traduit évidemment l'effet conjugué de l'altération phylliteuse et carbonatée, peut s'accompagner d'un apport ou d'un lessivage d'éléments chimiques.

Ainsi, les variations des principaux oxydes en fonctions des pertes au feu (fig 20) permettent de rendre compte du comportement des éléments chimiques au cours de l'altération. Dans ce sens, le diagramme SiO2-PF montre une diminution des teneurs en $\mathrm{SiO} 2$ corrélativement à l'augmentation de celle de pertes au feu, cette distribution des points représentatifs des échantillons analysés est sans doute une conséquence d'un hydrothermalisme responsable de la mobilité de la silice comme en témoigne la présence de filons, veines, et vacuoles remplis de quartz secondaire.

Le diagramme $\mathrm{CaO}$ - PF montre une corrélation négative marqué par une augmentation des teneurs en $\mathrm{CaO}$ pour des pertes au feu de plus en plus élevées, ceci peut être expliqué par la mobilité du calcium comme témoigne d'une part la calcitisation des plagioclases et de la mésostase et d'autre part, le remplissage des vacuoles, des veines et des filons de la calcite

Dans le diagramme de $\mathrm{Fe} 2 \mathrm{O} 3$ - $\mathrm{PF}$ une légère corrélation négative indique un lessivage du fer, comme le laisse prévoir l'oxydation des microfractures, l'hématisation et l'oxyde de fer dans cassures et le clivage dans les clinopyroxènes

Le diagramme de MgO- PF met en évidence une corrélation positive des représentatifs des roches basiques qui traduit un apport du magnésium, comme l'atteste la formation de la chlorite magnésienne au dépend des minéraux ferromagnésiens (chloritisation des clinopyroxène, dans les vacuoles et dans la mésostase

Le diagramme de $\mathrm{K} 2 \mathrm{O}$ vs $\mathrm{PF}$ met en évidence une corrélationnégative qui indique que les phénomènes d'altération s'accompagnent d'unlessivage de $\mathrm{K} 2 \mathrm{O}$.

Le $\mathrm{Na} 2 \mathrm{O}$ vs $\mathrm{PF}$ présente une très faible corrélation positive et pourraits'expliquer par une contribution du $\mathrm{Na}$ marquée par l'albitisation desplagioclases calciques. 
En consultant le diagramme $\mathrm{K} 2 \mathrm{O}$ - Na2O nous constatons que ces deux éléments sont liés par une corrélation négative si bien que les échantillons les plus potassiques étant les plus sodiques

Certes, cette évolution ne peut trouver une explication que dans les processus de différenciation magmatique, nous envisageons une métasomatose potassique couplée à un lessivage de la soude pour interpréter le comportement des alcalins dans roches magmatiques basiques du bassin de MBA
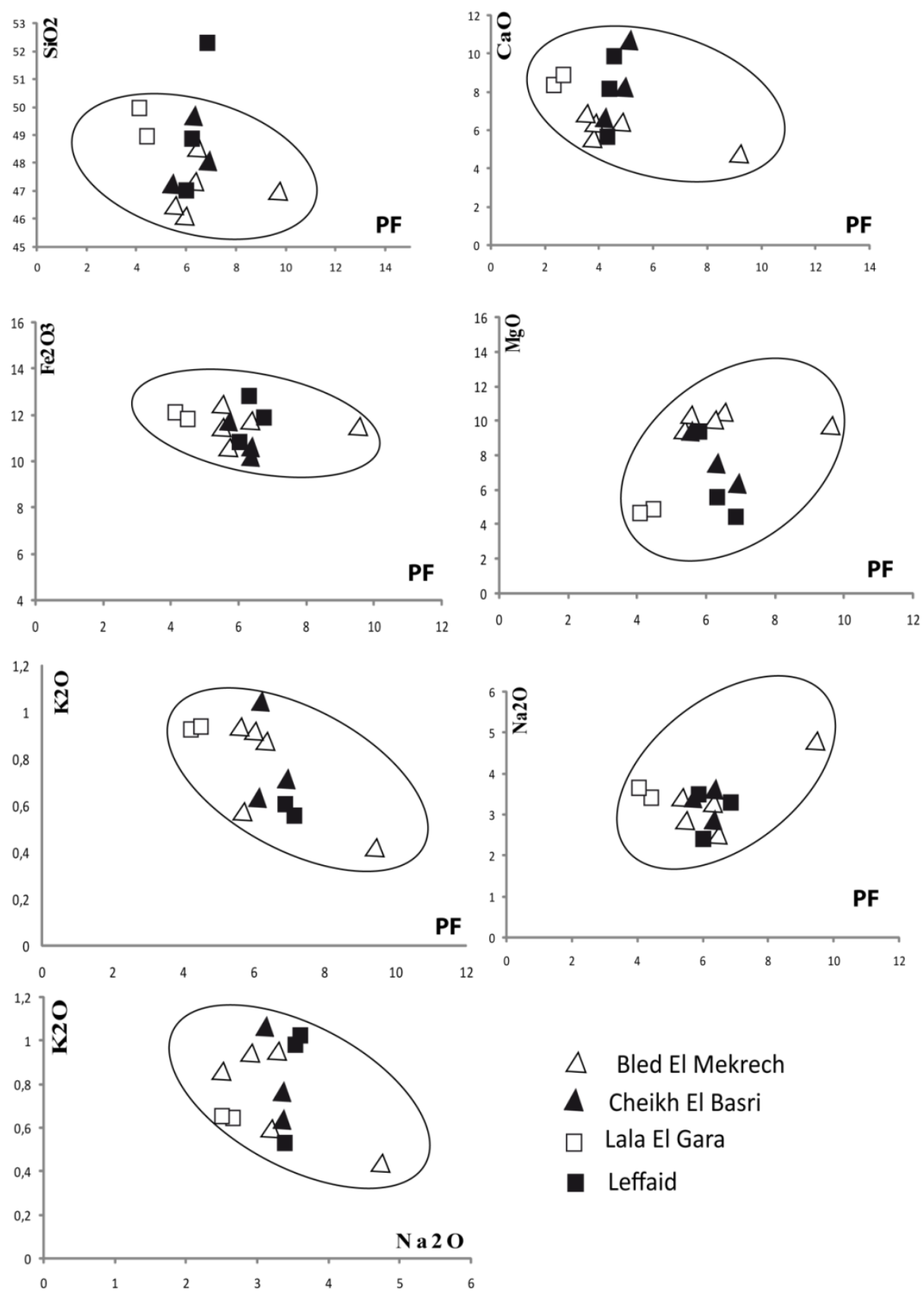

$\triangle$ Bled El Mekrech

$\Delta$ Cheikh El Basri

$\square$ Lala El Gara

- Leffaid

Fig. 20:-Variation de la composition chimique des éléments majeurs en fonction de la perte au feu 


\section{Conclusion générale:-}

Dans le but d'approcher les phénomènes d'altération dans les roches magmatiques basiques de Mechraa Ben Abbou nous étions amenés à suivre une démarche multiple structurale, pétrographique et géochimique. Ainsi, l'analyse structurale de quatre affleurements représentatifs a permis à travers des coupes réalisées sur le terrain que les contacts entre les différentes roches magmatiques et l'encaissant immédiat sont toujours parallèles à la stratification ce qui témoigne d'une mise en place de ces roches sous forme de sills.

L'étude pétrographique des roches magmatiques des quatre affleurements (Bled ElMekrech, Cheikh El Basri, Lala El Gara et Leffaid) a permis de remarquer que ces roches présentent des minéralogies comparables dominées par une paragénèse primaire constituée par une abondance de plagioclase suivie par le clinopyroxène et une paragenèse secondaire qui est surtout représentée par des minéraux d'altérations tels que la calcite, la chlorite, l'amphibole, et l'oxyde de fer.

Ces phénomènes d'altérations qui peuvent être liés aux circulations des fluides hydrothermaux se manifestent à l'échelle de l'affleurement et du banc par des filons de calcites, de quartz et d'ankérite et à l'échelle de la lame mince les roches magmatiques des quatre zones d'études montrent des transformations en calcites, en chlorites, en oxydes de fer et en quartz rarement au centre des sills, mais très intensément aux bordures figées. Les altérations se développent, aussi bienau niveau de la mésostase qui inclut localement des vacuoles millimétriquescolmatées par la calcite et la chlorite.

Les analyses géochimiques montrent que les altérations se traduisent par un changement dans le comportement des éléments chimiques qui témoigne de l'interaction entre les fluides hydrothermaux et les roches magmatiques étudiées. Ainsi, les diagrammes de variation des oxydes en fonction des pertes au feu indiquent un lessivage de $\mathrm{SiO}_{2}, \mathrm{CaO}, \mathrm{Fe}_{2} \mathrm{O}_{3}$ et de $\mathrm{K}_{2} \mathrm{O}$ et d'un apport enMgO et en $\mathrm{Na}_{2} \mathrm{O}$.

La combinaison des données structurales (filons plissées et filons cassées), la nature du remplissage minéral séquentiel avec la cristallisation de la chlorite (haute $\mathrm{T}^{\circ}$ ) et tardive de la calcite (basse $\mathrm{T}^{\circ}$ ) plaident pour deux phases d'altérations hydrothermales concordantes avec les deux phases tectoniques hercyniennes paroxysmale et tardive :

- Une phase I, caractérisée par un plissement P1 à l'échelle cartographique dont l'axe est orienté N20- N 30 avec un plongement de 10 à $20^{\circ}$ vers le Sud. C'est le synclinal plurikilométrique dit de la Gada Jennabia ;

- Une phase II, caractérisée par un plissement P2 détectable à l'échelle de l'affleurement métrique à décamétrique accompagnant les failles transverses N60-N80.

\section{References:-}

1. Aarab El M., (1995)- Genèse et différentiation d'un magma tholéitique en domaine extensif intracontinental: Exemple du magmatisme pré-orogénique des Jebilet (Maroc hercynien). Thèse ès Sciences, Université Cadi Ayad, Marrakech, 253 p.

2. Ben Abbou M., (2001)- Dynamique des bassins d'avant pays carbonifères, signatures tectoniques, sédimentaires et magmatiques de l'évolution de la chaine hercynienne du Mar oc central septentrional ; implication sur le modèle géodynamique de la chaine hercynienne. Thèse ès Sci., Univ Cadi Ayad. Marrakech, $313 \mathrm{p}$.

3. Ben Abbou M.; Soula J.C.; Brusset S.; Roddaz M.; N'tarmouchant A.; Driouch Y.; Christophoul F.; Bouabdelli M.; Majeste-Menjoulas C.; Beziat D.; Debat, P. \&Deramond, J. (2001)- Contrôle tectonique de la sédimentation dans le système de bassin d'avantpays de la Meseta marocaine. Comptes Rendus de l'Académie des Sciences de Paris, 332: 703-709.

4. El Kamel F. (2002)- Sédimentologie, magmatisme pré-orogénique et structuration du Paléozoïque des Rehamna et d'Ouled Abbou (Meseta occidentale, Maroc). These, Doctorat es-Sciences, Universite Hassan II, Casablanca, Maroc, $208 \mathrm{p}$.

5. Essaifi A., (1995)- Relations entre Magmatisme-de'formation et altérations hydrothermales: l'exemple des Jebilet centrales (hercynien, Maroc). Mémoires Géosciences Rennes 56, 340 p.

6. Gigout, M. (1951)-Etudes géologiques sur la Meseta marocaine occidentale. Notes \&Mém. Serv. géol. Maroc, $86,507 \mathrm{p}$.

7. Hughers C. (1973)-spilites, Kératophyres and igneous spectrum.Geol. Mag, 109 pp 513-527

8. Huvelin P. (1977)- Etude géologique et pétrologique du Massif hercyniendes Jebilet (Maroc). Notes et Mémoires du Service Géologique du Maroc,232b, 307 pp. 
9. Jenny P. (1974)- contribution à la géologie structurale des rehamna (Mesetamarocaine méridionale). thèse de 3ème cycle, univ. louis pasteur destrasbourg, $120 \mathrm{p}$.

10. Kharbouch F., (1982)- les laves Dévano-dinantiennes de la Meseta marocaine : étude pétro-géochimique et implication géodynamique. Thèse ès sci.,Univ de Bretagne occidentale, Brest, 366p.

11. Kharbouch, F., (1994)- Le volcanisme dévono-dinantien du massif central et de ma mesetaa marocaine. Bull Inst. Sci Rabat 1994. ํ⒙

12. Kholaiq M., Saber N., et Zahour G. (2015a)- Le bassin Dévono-Dinantien de Mechraa Ben Abbou: bassin pull apart sur décrochement dextre (Rehamna Septentrionaux, Maroc):European Scientific Journal, édition vol.11, No.21, 361-376;

13. Kholaiq M., Saber N., et Zahour G. (2015b)- Contribution à l'étude du magmatisme basique associé au Viséen supérieur du bassin hercynien de Mechraa Ben Abbou (Rehamna-Maroc):European Scientific Journal, édition vol.11, No.21, 204- 221;

14. Kholaiq M., Saber N., et Zahour G., Maacha L., EL Hadi H., Oukassou M. (2016). Tholeiitic volcanic rocks of the Mechraa Ben Abbou Basin (Northern Rehamna, Western Meseta, Morocco): Petrography, geochemistry and geodynamic setting. Geo-Temas, édition vol.16, 443-447

15. Lagarde J.L., (1987)- les plutons granitiques hercyniens marqueurs de la déformation crustale : exemple de la méseta marocaine. Thèse de Doctorat d'état. Rennes, 371p.

16. Michard, A. (1976)-Elémentsde géologie marocaine. notesmém. serv. géol. maroc, 252, 408 p.

17. Mrini, Z., Rafi, A., Duthou, J-L. et Vidal, P. (1992)- Chronologie Rb-Sr des granitoïdes hercyniens du Maroc: conséquences. Bulletin de la Société Géologique de France, 163: 281-291 pp.

18. Ntarmouchant A., Smaili H., Bento dos Santos T., Dahire M., Sabri K., Ribeiro M.L., Driouch Y., Santos R., Calvo R. (2016) New evidence of effusive and explosive volcanism in the Lower Carboniferous formations of the Moroccan Central Hercynian Massif: Geochemical data and geodynamic significance, Journal of African Earth Sciences 115, 218-233

19. Remmal T., El Kamel F., et Mohcine A., (1997)- le Viséen de Mechraa Ben Abbou (meseta occidentale, Maroc) : une structure en décrochement $\mathrm{N} 80^{\circ}$ associées à un magmatisme tholeitique d'intraplaque. Rev. Géologie méditerranéen, Tome XXIV No 4

20. Remmal T., (2000)- L'évolution tectono-magmatique intracontinentale du cycle hercynien. Etude du complexe magmatique du district d'El Hammam et de zone comparable pour le magmatisme pré-orogéniaque dans le Massif central et les Rehamna (Meseta occidentale marocaine). Thèse ès sci.,Univ. Hassan II Ain Chok Casablanca, 267p.

21. Roddaz M., (2000)- Magmatisme de bassin d'avant-pays : exemple du magmatisme hercynien de la Meseta occidentale (Maroc). Mémoire DEA, univ Paul Sabatier, Toulouse, 57p.

22. Saber N., (1989)- Evolution sédimentaire du bassin Devono-Dinantien de Mechraa Ben Abbou (Rehamna septentrionaux). Thèse de 3ème cycles. UnivCaddiAyyad, fac Sci Marrakech, 172P. 